\section{Accounting for all the factors}

\section{By Matthew Mikulski, Staff Writer}

Techniques for quantifying transcription factor activity have been limited to examining only a few targets at a time. Now, researchers at Attagene Inc. have published in Nature Methods an approach to simultaneously report on the activity of a panel of several dozen different transcription factors in cells. ${ }^{1}$ Genomics and transcriptomics service providers think the tool could have utility in toxicology studies while complementing existing techniques for profiling transcriptional activity within cells.

Many biological pathways, including those involved in inflammation, stress responses and cell-cycle control, converge on the activation of specific families of transcription factors (TFs). Thus, the activity of these TFs can be used to characterize and identify drug-induced or disease-associated cell phenotypes.

Until now, multiplex TF activity assays have been limited to protein-based reporting systems, which quantify the activity of endogenous TFs by measuring the protein products of genes that have been artificially introduced into cells. A key limitation is that the luciferases, fluorescent proteins and other reporter proteins only permit the study of the activity of three or four TFs at a time.

Early attempts to expand the bandwidth of the reporter-based assay by using mRNAs as reporter molecules failed because transcripts varying substantially in nucleotide sequence have different degradation rates, half-lives in cells and PCR amplification efficiencies.

By contrast, Attagene's mRNA-based technique achieves relatively uniform cellular processing and amplification because all reporter transcription units (RTUs) are practically identical in sequence.

Each RTU consists of a DNA binding site that is unique to a particular TF or TF family. The site is located upstream from a reporter sequence that codes for an mRNA about 800 base pairs long. The nucleotide sequences of these RTUs differ only in the location of an endonuclease recognition site, which is placed at a unique interval for each RTU.

"When they get degraded, they do so at the same rate; when they get amplified, they do so at the same rate," said Sergei Makarov, president and CEO of Attagene and a senior author on the paper.

After reverse transcriptase PCR converts the RTUs into DNA and amplifies the signals, the reporters are labeled with fluorescent protein and treated with endonuclease. This yields a spectrum of DNA fragments of different lengths that each correspond to a different RTU and can thus be used to determine the activity of each TF.

In several experiments using HepG2 liver cancer cells, activity levels reported by a 43-member multiple RTU (MRTU) panel deviated by $1-14 \%$. The authors said that performance was comparable to research tools that quantify gene expression.

Assay output was not affected by the quantity of DNA used to transfect cells with the MRTU library, the quality of mRNA acquired for analysis or the number of PCR cycles. Thus, Makarov said, the data suggest the assay can generate standardized results.

Indeed, the assay reliably detected increases in TF activity resulting from compounds with known transcriptional effects. Moreover, the researchers showed that two different types of colorectal cancer cell lines and four different types of breast cancer cell lines had distinct TF activity profiles.

Philip Gregory, VP of research at Sangamo BioSciences Inc., told $S c i B X$ the MRTU assay could allow biotech and pharma companies to look at dozens of pathways that are of "incredible interest," including those involved in xenobiotic response, cell-cycle control and hormone receptor pathways that drive differentiation.

Sangamo develops zinc finger DNA-binding protein transcription factors (ZFP) that regulate gene expression. Because the company can design ZFPs with specificities down to a single base pair, Sangamo can use gene expression arrays to confirm on-target activity. ${ }^{2}$

With the MRTU assay, Gregory noted, "you don't have to know what the downstream effects are; all you are asking is whether that

factor itself is active."

Gregory said the assay could be useful for small-molecule developers who want to quantify the effects of their compounds. In addition, he suggested the assay could be applied to drug discovery. Because the expression profiles of different cancer cells are distinct, he said, there is "the hope of finding similarities or differences that can be exploited."

Makarov said Attagene is building a database of TF activity profiles associated with approved drugs as well as failed drug candidates. The company already provides screening services to undisclosed pharma companies.

Attagene has a high-throughput robotics facility that can screen hundreds of compounds in a day. The company's technology was selected as one of eight to be used in ToxCast, a $\$ 120$ million predictive toxicology project launched last year by the U.S. Environmental Protection Agency.

Ray Blanchard, senior director of technology development at microarray service and supply company SuperArray Bioscience Corp., agreed that profiling TF activity using something such as the MRTU assay could be useful in both toxicology and early drug discovery studies. For basic research, however, he thinks the MRTU assay would need to work in concert with existing genomics and transcriptomics tools, 


\section{TARGETS \& MECHANISMS}

such as those based on chromatin immunoprecipitation (ChIP).

ChIP chemically cross-links DNA-binding proteins with the segment of chromatin they interact with. Within a chosen genomic region, the genes with which the proteins associate can be identified and their frequency of association derived.

Mary Harper, CSO of ChIP assay company Genpathway Inc., agreed that combinations of tools are likely to be used in basic research. For example, she said the MRTU assay could give an overview of the genes that are upregulated in specific disease states, after which ChIP could identify exactly which genes are recruiting the TFs.

Sergei Romanov, VP and CSO at Attagene and first author on the paper, told $S c i B X$ that ChIP is unable to show direct relationships between TF binding and gene expression because endogenous mRNAs are often differentially regulated through post-transcriptional mechanisms.

Although the MRTU assay measures mRNA produced by transcriptionally active TFs, Makarov said DNA-binding assays such as ChIP are not always sufficient to enable measurement of a given TF's modulatory effects. Nuclear receptors provide a case in point, he said. These DNAbinding, transcription-regulating proteins "sit on DNA all the time-so if you measure binding, it doesn't tell you much."

Claus Kremoser, CEO of nuclear receptor screening and therapeutics company Phenex Pharmaceuticals AG, agreed the activity of nuclear receptors and even some TFs is beyond the scope of ChIP.

On the other hand, the MRTU assay does have limitations. Harper said each RTU used in Attagene's assay incorporates only a single unique binding site - the given TF's consensus binding sequence-in its promoter region. TFs have been shown to bind to nonconsensus DNA sequences, and any transcriptional activity that may result would not be reflected in the MRTU assay.

Kremoser agreed the MRTU assay represents a considerable simplification of what is actually going on in cells. He said routine use will reveal whether the assay "can really reflect the complex transcriptional activation status that is exerted by an individual transcription factor."

Because hundreds of distinct transcripts can be built from a single reporter template, Makarov said it should be straightforward to expand the MRTU panel to cover all of the several hundred estimated TF families in the human genome. "Within two years, we will be able to embrace the whole 'TFome"' he said.

Other planned improvements include getting the system to work in the mouse heart and brain. Makarov said the company already has reliable MRTU assay function in mouse liver and expects to submit a manuscript by year end.

Attagene offers high-throughput screening services covering a panel of 46 transcription factors and 20 nuclear receptors and also markets a reagent kit for academic researchers. The first kit covers a panel of $30 \mathrm{TFs}$, with subsequent releases tentatively planned to cover 50 and 70 TFs.

\section{REFERENCES}

1. Romanov, S. et al. Nat. Methods; published online Feb. 24, 2008; doi:10.1038/nmeth.1186

Contact: Sergei Makarov, Attagene Inc., Research Triangle Park, N.C. e-mail: smak@attagene.com

2. Tan, S. et al. Proc. Natl. Acad. Sci. USA 100, 11997-12002 (2003)

COMPANIES AND INSTITUTIONS MENTIONED

Attagene Inc., Research Triangle Park, N.C.

Genpathway Inc., San Diego, Calif.

Phenex Pharmaceuticals AG, Ludwigshafen, Germany

Sangamo BioSciences Inc. (NASDAQ:SGMO), Richmond, Calif. SuperArray Bioscience Corp., Frederick, Md. 